\title{
Optimal Placement of Access Point in WLAN Based on a New Algorithm
}

\author{
S. Kouhbor, Julien Ugon, Alex Kruger, Alex Rubinov \\ School of Information Technology and Mathematical Sciences \\ University of Ballarat, Ballarat, Vic 3353 \\ skouhbor@students.ballarat.edu.au
}

\begin{abstract}
When designing wireless communication systems, it is very important to know the optimum numbers and locations for the access points (APS). The impact of incorrect placement of APs is significant. If they are placed too far apart, they will generate a coverage gap, but if they are too close to each other, this will lead to excessive co-channel interferences. In this paper we describe a mathematical model developed to find the optimal number and location of APs. To solve the problem, we use the Discrete Gradient optimization algorithm developed at the University of Ballarat. Results indicate that our model is able to solve optimal coverage problems for different numbers of users.
\end{abstract}

\section{Introduction}

The primary goal of Wireless Local Area Network (WLAN) deployment is to provide total coverage for all users in the design area. In other words, how and where to locate the APs, so that the primary goal of deployment is satisfied. The location of the APs determines the standard of coverage of the design area. Coverage is determined by the number of APs that are placed in the design area. Placing too many APs increases the cost of deployment and placing a few will lead to coverage gaps which prevents users from having access to their data through APs.

The method used by network operators to find the placement of AP is based on the RF (radio frequency) site survey. This involves network designer going around the facility and measuring the RF signal strengths at various locations using software running on a laptop or PDA (Personal Digital Assistant). This software detects places that are suitable for APs to be installed based on signal strength, noise levels, and signal quality. These measurements have to be repeated many times to ensure reliable results. However, this method is expensive and its results are not very reliable due to characteristics of the building and location of the users that can change with time [1].

Another approach that is used by researchers [1-13] to find the optimal placement of APs is through the use of optimization techniques. Most of the authors [1-8] use discrete mathematical models to find the position of APs. In this case, the design area is divided into rectangles (grids). APs are only allowed to be placed in the centers of the rectangles. To obtain satisfactory results, the size of the grid must be sufficiently small. However, in this case, the dimension of the problem can be very high. For this reason some authors [9-11] prefer continuous mathematical models. Others $[12,13]$ have tried to compare their results using both methods.

The model investigated in the current paper is also based on applying continuous optimization techniques with no restrictions on the position of APs.

It should be noted that there are different approaches to solve optimization problem in hand. In all these approaches there are two types of variables in the model: integer variables and continuous ones. Continuous variables describe the location of APs whereas integer variables describe the membership degree of receivers to clusters (group of users/receivers congregating in one area and using the same AP). Since each receiver can belong only to one cluster, integer variables can attain values of 0 and 1 only. As a result we get a mixed integer nonlinear programming problem. It is well known that such problems are difficult to solve in many situations. A nonsmooth optimization approach described in this paper allows one to exclude integer variables, to reduce significantly the number of variables in the optimization problem and to replace the mixed integer nonlinear programming problem with a continuous nonlinear programming problem.

This paper is organized as follow: Section 2 shows the model notation. Section 3 presents the mathematical model. Method for the solution of the problems is described in Section 4. The testing method is described in Section 5. Results and the effect of AP parameters on coverage are discussed in Section 6. The 
final section summarizes the paper and discusses future research.

\section{General notations} used:

Throughout this paper the following notations are

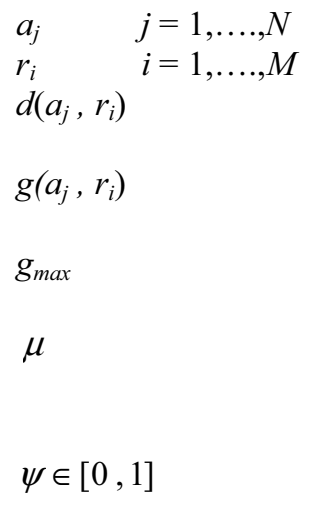

Access point (AP)

Receiver/user

Distance between AP and

receiver

Path loss from $i^{\text {th }}$ user to access point $j$

Maximum tolerable path loss

Penalty parameter for

violating the maximum

tolerable path loss

Convex combination

coefficient used to define

the sum and max objective

function

$P_{t} \quad$ Transmit power

$P_{r} \quad$ Received power

$R_{t h} \quad$ Receive threshold

$a_{p} \quad$ Position of AP

All points in the design area are represented by their coordinates (either two - on the plane or three in the three dimensional space). We assume the distance function to be Euclidean, hence on the plane, the distance $(d)$ between an AP $a_{j}$ and a receiver $r_{i}$ is given by:

$$
d\left(a_{j}, r_{i}\right)=\sqrt{\left(r_{i}^{1}-a_{j}^{1}\right)^{2}+\left(r_{i}^{2}-a_{j}^{2}\right)^{2}}
$$

where $a_{j}=a_{j}\left(a_{j}{ }^{1}, a_{j}{ }^{2}\right)$, and $r_{i}=r_{i}\left(r_{i}{ }^{1}, r_{i}{ }^{2}\right)$.

\section{Optimization model}

Some researchers $[1-7,9,12]$ have used path loss while others $[10,11,13]$ have used power at the receiver to develop their optimization problem. In this paper we follow the first approach to find the optimal placement of APs. Path loss model is the core of the signal coverage calculation for any environment [14]. It describes the loss of signal strength due to distance and obstacles between transmitter and receiver. Path loss models can be used to calculate the coverage area of AP and maximum distance between the two terminals in an ad hoc network.

\subsection{Model description}

The objective function based on path losses can be developed in different formats such as the average path loss, the maximal path loss, the sum of squared path losses, and a convex combination (an approach used to combine two extreme cases) of the average and the maximal path losses with different coefficients. In this paper, we consider the last approach.

$$
F_{1}\left(a_{1}, \ldots, a_{N}\right)=\frac{1}{M} \sum_{i=1}^{M} \min _{j} g\left(a_{j}, r_{i}\right) .
$$

The above objective function provides coverage for the users in the design area. The drawback with this objective function is that it might ignore a few remotely located users. The maximal path loss over the design area is given by:

$$
F_{2}\left(a_{1}, \ldots, a_{N}\right)=\max _{i} \min _{j} g\left(a_{j}, r_{i}\right) .
$$

The disadvantage of this objective function $\left(F_{2}\right)$ is that it concentrates on the worst case situation, at the expense of the overall average weighted cover. The total objective function can be taken as a convex combination of $F_{1}$ and $F_{2}$, controlled by a coefficient $\psi \in[0,1]$ :

$$
\begin{gathered}
F_{3}=\psi_{F_{1}}+(1-\psi) F_{2}, \\
F_{3}\left(a_{1}, \ldots, a_{N}\right)=\psi\left(\frac{1}{M} \sum_{i=1}^{M} \min _{j} g\left(a_{j}, r_{i}\right)\right) \\
+(1-\psi)\left(\max _{i} \min _{j} g\left(a_{j}, r_{i}\right)\right) .
\end{gathered}
$$

The objective function (1) is minimized subject to the following constraints:

$$
\min _{j} g\left(a_{j}, r_{i}\right) \leq g_{\max } \quad \forall i=1, \ldots ., M .
$$

These constraints state that path loss is evaluated against the maximum tolerable path loss $g_{\max }$. This ensures that the quality of coverage at each receiver location is above the given threshold. This given value, $g_{\max }$ can be calculated by subtracting the receiver threshold from the transmitter power.

$$
g_{\max }=P_{t}-R_{t h} \cdot
$$


The above inequality (2) can be expressed in the equality form as:

$$
\left(\min _{j} g\left(a_{j}, r_{i}\right)-g_{\max }\right)^{+}=0,
$$

where $(\alpha)^{+}=\max (\alpha, 0)$.

The above problem of minimizing (1) subject to (2) can be converted into the unconstrained one by using the penalty approach. If the path loss threshold $\left(g_{\max }\right)$ at a receiver location is violated, a penalty term depending on a parameter $\mu$ will be added. Therefore, our fourth function can be written as:

$$
F_{4}\left(a_{1}, \ldots, a_{N}\right)=\mu \sum_{i=1}^{M}\left(\min _{j} g\left(a_{j}, r_{i}\right)-g_{\max }\right)^{+} .
$$

The total objective function is the sum of $F_{3}$ and $F_{4}$ :

$$
\begin{aligned}
& F_{5}\left(a_{1}, \ldots, a_{N}\right)=\psi\left(\frac{1}{M} \sum_{i=1}^{M} \min _{j} g\left(a_{j}, r_{i}\right)\right) \\
& +(1-\psi)\left(\max _{i} \min _{j} g\left(a_{j}, r_{i}\right)\right) \\
& +\mu \sum_{i=1}^{M}\left(\min _{j} g\left(a_{j}, r_{i}\right)-g_{\max }\right)^{+}
\end{aligned}
$$

\subsection{Path loss model}

In general the power received by an antenna that is separated from the transmitting antenna by the distance $d$ in free space is given by $[14,15]$ :

$$
P_{r}\left(a_{j}, r_{i}\right)=\frac{P_{t} G_{t} G_{r} \lambda^{2}}{(4 \pi)^{2} d\left(a_{j}, r_{i}\right)^{2}}
$$

where $P_{t}$ is the transmitted power, $G_{t}$ and $G_{r}$ are the transmitter and receiver antenna gain, $d$ is the distance between transmitter and receiver, and $\lambda=c / f$ is the wavelength of the carrier frequency, $c$ is the speed of light ( $3 \times 10^{8}$ meter per second) and $f$ is the frequency of radio carrier in hertz. The path loss, which represents signal attenuation between the transmitted and the received power and is measured in $\mathrm{dB}$ (decibels), in free space environments, is given by [15]

$$
g\left(a_{j}, r_{i}\right)[d B]=-10 \log \left[\frac{G_{t} G_{r} \lambda^{2}}{(4 \pi)^{2} d\left(a_{j}, r_{i}\right)^{2}}\right] .
$$

The above equation does not hold when points $a_{j}$ and $r_{i}$ are very close to each other. Therefore, large scale propagation models use a close-in distance, $d_{0}$, which is known as the received power reference distance point. Therefore, path losses at reference distance assuming transmit and receive antenna with unity gain as described in [14-16] can be calculated from:

$$
g\left(a_{j}, r_{i}\right)=g\left(d_{0}\right)[d B]=20 \log \frac{4 \pi d_{0} f}{c} .
$$

Therefore, path loss function in free space at a distance greater than $d_{0}$ is given by [14-16]:

$$
g\left(a_{j}, r_{i}\right)[d B]=g\left(d_{0}\right)[d B]+10 \log \left(\frac{d\left(a_{j}, r_{i}\right)}{d_{0}}\right)^{2} .
$$

\section{Solution of the problem (1)-(2)}

The objective function (1) is nonsmooth and nonconvex and it has many local minima. When the number of AP is large, we get a large scale global optimization problem. However, traditional global optimization methods cannot be directly applied to it.

Computation of subgradients of the function $F_{5}$ is a very difficult task. Therefore, methods requiring subgradient evaluation at each iteration cannot be effective.

Direct search methods seem to be the best option for solving problem (1)-(2). However, for many such methods the number of variables and/or constraints, which can be efficiently handled, is restricted. For example, one of the most efficient direct search methods - the Powell method [17] performs well when the number of variables is less than 20. However, in the problem under consideration, the number of AP can be large, therefore, the number of variables can be much more than 20. (The number of variables is the number AP multiplied by the number of coordinates. For example, in case of two APs in three dimensional space, the number of variables is 6 .)

We use the Discrete Gradient method to solve Problem (1)-(2). The description of this method can be found in $[18,19]$. This is a derivative-free method. The Discrete Gradient method consists of two main steps: the computation of a descent direction which is reduced to a certain quadratic programming problem 
and a line search. We use so called Armijo-type line search in this method. As it is shown in [19], computation of the descent direction is a terminating process: after a finite number of steps the algorithm either computes the descent direction or finds out that the current iteration is a stationary point. The Discrete Gradient method is efficient for solving large scale nonsmooth optimization problems. It is suitable for finding local minimizers in Problem (1)-(2). This algorithm allows a continuous search to find the optimal placement of APs meaning no restriction is placed on their positions.

\subsection{Finding the number and location of APs}

Initially we set the number of AP to $1: N=1$; then the necessary number of APs is found through the following steps:

1. Try to solve Problem (1)-(2);

2. If the solution exists, then $N$ is the desired number;

3. Otherwise, $N$ is increased by $1: N=N+1$;

4. Go to step 1.

\section{Method of testing}

Two simple cases without obstacles were considered for conducting the test in order to examine the model. In the first case, the design area is $1000 \mathrm{~m} \mathrm{x}$ $1500 \mathrm{~m}$ and has 75 users. In the second case, random number generator is used to generate 80 users in different parts of the area which is extended to $1500 \mathrm{~m}$ x $2000 \mathrm{~m}$. The two cases are shown in Figures 1a and 1b. The specifications of two models of APs developed by Cisco (Aironet 1200 series and Aironet 340 series) $[20,21]$ and IEEE 802.11b standard [22] are used to test the model. This gives us the opportunity to examine the model with extreme (very high to very low) values of $P_{t}$ and $R_{t h}$ to find the effect of AP parameters on the capacity that is going to be considered for further development of the research.

\section{Results}

\subsection{Case one: design area $1000 \mathrm{~m} \times 1500 \mathrm{~m}$}

In order to examine the behavior of our model, we chose different values for the coefficient (balancing parameter) $\psi$. We shall present here the results for the three cases $\psi=0, \psi=1$ and $\psi=0.5$ with some of the values of $P_{t}$ and $R_{t h}$. It should be noted that in the developed model, the aim is to provide only the coverage for the users.

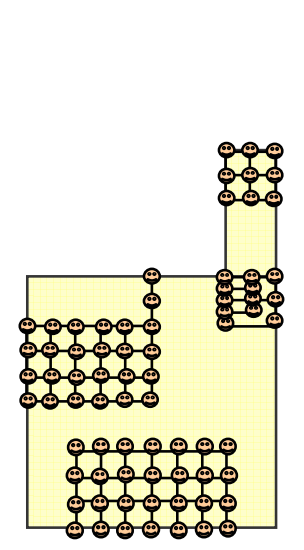

Figure 1a. Position Of 75 users

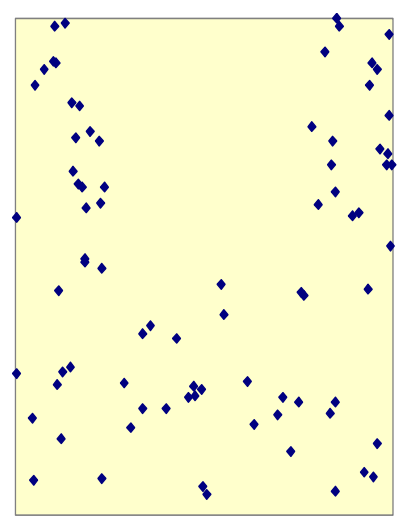

Figure 1b. Position of 80 80 users
Case 1.1: $\psi=0$. Table 1 shows the optimal placement of AP for some of the values of $P_{t}$ and $R_{t h}$ when $\psi=0$.

When $\psi=0$, the average path loss is ignored and emphasis is placed on the maximal path losses. For high value of $P_{t}$ and low value of $R_{t h}$, one AP placed in the middle of the area is able to cover all the users. In this case all users have equal access to AP. As these parameters are changing, the position and number of APs are changing as well. In the case when $P_{t}$ is 20 $\mathrm{dBm}$ and $R_{t h}$ is $-76 \mathrm{dBm}$, two APs are required to cover the users. Figure 2 shows the coverage of the users. Figure 3 shows that when $P_{t}$ decreases to $15 \mathrm{dBm}$ and $R_{t h}$ is kept at $-76 \mathrm{dBm}$, the required number of APs is increased to four. This is due to distance between AP and user that is decreasing. This effect is more noticeable when $P_{t}$ is kept at $15 \mathrm{dBm}$ and $R_{t h}$ is further increased. For example, figure 4 shows that thirteen APs are required to cover users when $P_{t}$ is kept at 15 $\mathrm{dBm}$ and $R_{t h}$ is increased to $-70 \mathrm{dBm}$.

Case 1.2: $\psi=1$. Table 2 shows the optimal placement of APs for some of the values of $P_{t}$ and $R_{t h}$ when $\psi=1$.

When $\psi=1$, the average path loss over the design area is minimized while satisfying the worse user condition is ignored. Comparing the results with Table 1 , in this case, for large values of $P_{t}$ and small value of $R_{t h}$ such as $P_{t}=20 \mathrm{dBm}$ and $R_{t h}=-85 \mathrm{dBm}$, the AP is placed on the top corner of the design area. Figure 5 shows that when $P_{t}$ decreases to $15 \mathrm{dBm}$ and $R_{t h}$ increases to $-70 \mathrm{dBm}$, the obtained number of APs increases to thirteen. This is the same as case 1.1 , but the position of APs is changed. Results indicate that in all cases the coverage of all users is assured.

Results obtained for other values of $\psi$ such as 0.1 to 0.9 indicate that for low values of $R_{t h}(\leq-85)$, the 
position and number of APs is the same as when $\psi=$ 1. When $R_{t h}$ is increasing, number of APs obtained is the same as case 1.1 and case 1.2 , but their positions are different. Table 3 shows results for some of the values of $P_{t}$ and $R_{t h}$ when $\psi=0.5$.

\subsection{Case 2: design area $1500 \mathrm{~m} \times 2000 \mathrm{~m}$}

Case 2.1: $\psi=\mathbf{0}$. In order to make sure that the model can respond to different types of environment, the area is extended to $1500 \mathrm{~m} \mathrm{x} 2000 \mathrm{~m}$ in a $\mathrm{U}$ shape format and random number generator is used to distribute users in all parts of the area.

The placement of AP for large values of $P_{t}$ and $R_{t h}$ of less than $-89 \mathrm{dBm}$ is the same as case 1.1 i.e. the AP is placed in the middle of the area. As $P_{t}$ decreases and $R_{t h}$ increases, more APs are needed to cover the users compared to case 1.1 due to the large size of design area and the method of distribution of users. Figure 6 shows the position of APs and coverage of users when $P_{t}=20 \mathrm{dBm}$ and $R_{t h}=-76 \mathrm{dBm}$.

Case 2.2: $\psi=1$. The obtained results are similar to the case 1.2. However, the number of APs for low values of $P_{t}$ and high values of $R_{t h}$ is increased.

\subsection{Processing time}

Condition of experiments. Tests were conducted on a PC that is equipped with Pentium (R) 4 processor, CPU of $3 \mathrm{GHz}$, and $496 \mathrm{MB}$ of RAM. Codes are written in $\mathrm{C}++$.

Due to efficiency of the Discrete Gradient algorithm, the processing time for satisfying the objective function is very short. Figure 7 shows that it takes 2:11:55 seconds to find the number and location of 49 APs (1:46:47 seconds for finding the number of APs and 25:8 seconds for finding their locations) when there are 80 users in the design area, $\psi=0, P_{t}=20$ $\mathrm{dBm}$, and $R_{t h}$ is changing from -89 to $-55 \mathrm{dBm}$. Using the same criteria, the processing time for one AP was 0.46 seconds.

\section{Conclusion}

This paper investigates allocation problems with objective functions based on minimizing the average path loss received over the entire design area and maximum path loss received by any receiver. The model described above can be used for finding the optimal placement of APs while covering as many users as possible. We used the Discrete Gradient
Table 1. Optimal placement of APs for 75 users when $\psi=0$

\begin{tabular}{|c|c|c|c|}
\hline $\begin{array}{c}P_{t} \\
(\mathrm{dBm})\end{array}$ & $\begin{array}{c}R_{t h} \\
(\mathrm{dBm})\end{array}$ & $\underset{(\mathrm{dB})}{g}$ & $a_{p}$ \\
\hline 20 & $\leq-85$ & 98.6 & {$[600,750]$} \\
\hline 20 & -76 & 95.3 & {$[500,500][1000,1483]$} \\
\hline 20 & -70 & 89.3 & $\begin{array}{l}{[550.3,150][860.5,1244.2]} \\
{[250,650][1000,800]} \\
{[450,150][500,1000]}\end{array}$ \\
\hline 17 & $\leq-85$ & 98.6 & {$[600,750]$} \\
\hline 17 & -76 & 92.7 & $\begin{array}{l}{[496,304][751,1150.6]} \\
{[0,800]}\end{array}$ \\
\hline 17 & -70 & 86.6 & $\begin{array}{l}{[650,150][813,900][150,} \\
650][950,1450][350,150] \\
{[517,677][500,1000]}\end{array}$ \\
\hline 15 & -76 & 109 & $\begin{array}{l}{[500,185][749.7,1249.7]} \\
{[195.7,772.4][1000,800]}\end{array}$ \\
\hline 15 & -70 & 84.2 & $\begin{array}{l}{[583.7,139][873,900]} \\
{[100,672.6][910.8,1418]} \\
{[300,127.5][388,836.2]} \\
{[529.4,603][739,150]} \\
{[300,500][0,500][200,300]} \\
{[400,300][500,1000]}\end{array}$ \\
\hline
\end{tabular}

Table 2. Optimal placement of APs for 75 users when $\psi=1$

\begin{tabular}{|c|c|c|c|}
\hline $\begin{array}{c}P_{t} \\
(\mathrm{dBm})\end{array}$ & $\begin{array}{c}\boldsymbol{R}_{t h} \\
(\mathbf{d B m})\end{array}$ & $\begin{array}{c}g \\
(\mathrm{~dB})\end{array}$ & $a_{p}$ \\
\hline 20 & $\leq-85$ & 98.3 & {$[999.5,1499]$} \\
\hline 20 & -76 & 89.5 & {$[499,500][999.5,1499]$} \\
\hline 20 & -70 & 83.1 & $\begin{array}{l}{[576,150][861.8,1264]} \\
{[245.4,680.4][999,800.7]} \\
{[445,150][500,999]}\end{array}$ \\
\hline 17 & $\leq-89$ & 98.4 & {$[999.5,1499]$} \\
\hline 17 & -85 & 94.1 & {$[800,999]$} \\
\hline 17 & -76 & 86.9 & $\begin{array}{l}{[401,299][789.3,1109]} \\
{[0.7,799.3]}\end{array}$ \\
\hline 17 & -70 & 80.5 & $\begin{array}{l}{[647,151][801,900][163,} \\
652][949,1448.7][364,150] \\
{[499,699][500,999]}\end{array}$ \\
\hline 15 & -76 & $\begin{array}{c}103 . \\
3\end{array}$ & $\begin{array}{l}{[500,186.4][750,1249.7]} \\
{[198,769.7][999.5,800.8]}\end{array}$ \\
\hline 15 & -70 & 74.6 & $\begin{array}{l}{[580,124][899,901][100,} \\
701][901,1400][300,99] \\
{[400,799][499,600][729,} \\
150][300,501][.7,501][201 \\
, 299][401,299][500,999]\end{array}$ \\
\hline
\end{tabular}

algorithm to solve the problem. Results obtained show that the value of the parameter $\psi \in[0,1]$ has an effect on the location of APs. It can be used to find an intermediate solution between the two cases in the mathematical model. It is observed that the size of the design area and the number of users and their locations 
have an effect on the location and the number of APs needed to cover users. It is noticed that path loss increases as a function of distance between transmitter and user. This shows that the method confirms the expected behavior of attenuation. The processing time was very short due to efficiency of software.

Further work will involve developing model based on distances and limiting number of users per an AP in order to increase the capacity for each user. Obstacles will be included in the mathematical model presented in this paper and test will be conducted.

Table 3. Optimal placement of APs for 75 users when $\psi=0.5$

\begin{tabular}{|c|c|c|c|}
\hline $\begin{array}{c}P_{t} \\
(\mathrm{dBm})\end{array}$ & $\begin{array}{c}R_{\text {th }} \\
(\mathbf{d B m})\end{array}$ & $\underset{(\mathrm{dB})}{g}$ & $a_{p}$ \\
\hline 20 & $\leq-85$ & 101.5 & {$[999.5,1499]$} \\
\hline 20 & -76 & 92.53 & {$[499,500][999.8,1499]$} \\
\hline 20 & -70 & 85.59 & $\begin{array}{l}{[601,115][894,1303][246,} \\
643][999,801][399,200] \\
{[500,999]}\end{array}$ \\
\hline 17 & $\leq-89$ & 101.5 & {$[999.5,1499]$} \\
\hline 17 & -70 & 83.77 & $\begin{array}{l}{[646,150][801,900][162,} \\
651.8][949.7,1449][363, \\
150.4][499,700][500,999]\end{array}$ \\
\hline 15 & -70 & 79.32 & $\begin{array}{l}{[551,150][899,901][100,} \\
701][901,1400][300,99] \\
{[400,799][499,600][750,} \\
150][300,501][.7,501][20 \\
1,299][401,299][500,999]\end{array}$ \\
\hline
\end{tabular}

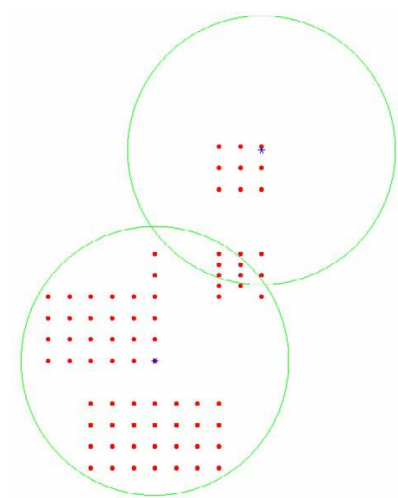

Figure 2. Coverage of 75 users when $\boldsymbol{P}_{t}=20 \mathrm{dBm}, \boldsymbol{R}_{t h}=-76 \mathrm{dBm}$, and $\psi=0$

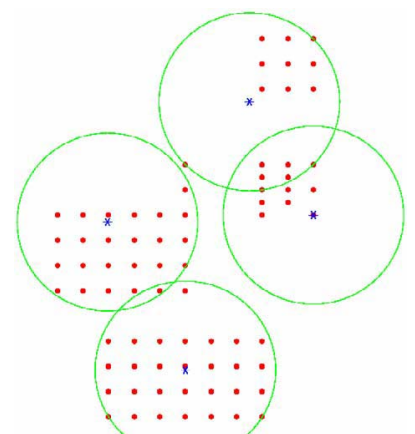

Figure 3. Coverage of 75 users when $P_{t}=15 \mathrm{dBm}, R_{t h}=-76 \mathrm{dBm}$, and $\psi=0$

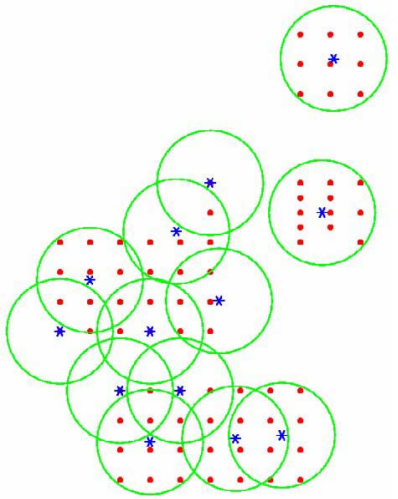

Figure 4. Coverage of 75 users when $P_{t}=15 \mathrm{dBm}, \boldsymbol{R}_{t h}=-70 \mathrm{dBm}$, and $\psi=0$

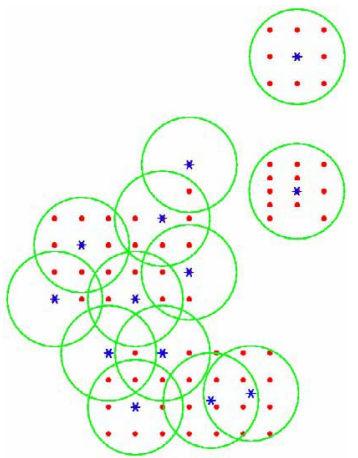

Figure 5. Coverage of 75 users when $P_{t}=20 \mathrm{dBm}, \boldsymbol{R}_{t h}=-70 \mathrm{dBm}$ and $\psi=1$

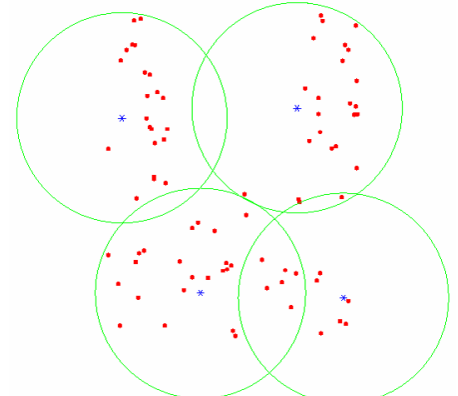

Figure 6. Coverage of 80 users when $P_{t}=20 \mathrm{dBm}, \boldsymbol{R}_{t h}=-76 \mathrm{dBm}$, and $\psi=0$ 


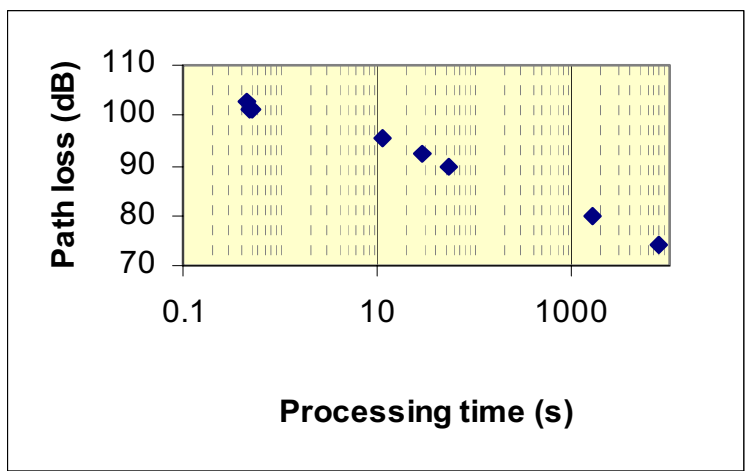

Figure 7. Processing time verses objective function (path loss)

\section{Acknowledgment}

The authors would like to thank Dr. Adil Bagirov for his valuable comments and suggestions.

\section{References}

[1] M. D. Adickes, R. E. Billo, B. A. Norman, S. Banerjee, B. O. Nnaji, and J. Rajgopal, "Optimization of Indoor Wireless Communication Network Layouts," IIE Transactions, vol. 34, pp. 823 - 836, 2002.

[2] M. Kamenetsky and M. Unbehaun, "Coverage Planning for Outdoor Wireless LAN Systems," presented at IEEE International Zurich Seminar on Broadband Communications, Sweden, 2002.

[3] K.-S. Tang, K.-F. Man, and S. Kwong, "Wireless Communication Network Design in IC Factory," IEEE Transaction on Industrial Electronics, vol. 48, pp. 452 - 458, 2001.

[4] M. Unbehaun and M. Kamenetsky, "On the Deployment of Picocellular Wireless Infrastructure," IEEE Wireless Communication Magazine, vol. 10, pp. 70 - 80, 2003.

[5] B.-S. Park, J.-G. Yook, and H.-K. Park, "The Determination of Base Station Placement and Transmit Power in an Inhomogeneous Traffic Distribution for Radio Network Planning," presented at IEEE 56th Vehicular Technology Conference, 2002.

[6] H. R. Anderson and J. P. McGeehan, "Optimizing Microcell Base Station Locations Using Simulated Annealing Techniques," Proceedings of the 44th Vehicular Technology, pp. 858 - 862, 1994.

[7] H. D. Sherali, C. M. Pendyala, and T. S. Rappaport, "Optimal Location of Transmitters for Micro-Cellular Radio Communication System Design," IEEE Journal on Selected Areas in Communications, vol. 14, pp. 662 - 673, 1996.

[8] Y. Lee, K. Kim, and Y. Choi, "Optimization of AP Placement and Channel Assignment in Wireless LANs," presented at Proceedings of the 27th Annual IEEE Conference on Local Computer Networks (LCN'02), 2002
[9] S. Kouhbor, J. Ugon, A. Kruger, A. M. Rubinov, and P. Branch, "A New Algorithm for the Placement of WLAN Access Points Based on Nonsmooth Optimization Technique," presented at 7th International Conference on Advanced Communication Technology (IEEE/ICACT2005), February 2005, PP. 352-357

[10] S. Fortune, D. Gay, B. Kernigban, O. Landron, R. Valenzuela, and M. Wright, "Wise Design of Indoor Wireless System: Practical Computation and Optimization," in IEEE Computational Science and Engineering, 1995, pp. 58 -68.

[11] S. Kouhbor, J. Ugon, A. Kruger, A. Rubinov, and P. Branch, "Optimization in Wireless Local Area Network," presented at 6th International Conference on Optimization: Techniques and Applications (ICOTA), Ballarat, Australia, 2004.

[12] Z. Ji, T. K. Sarkar, and B.-H. LI, "Methods for Optimizing the Location of Base Stations for Indoor Wireless Communications," IEEE Transactions on Antennas and Propagation, vol. 50, pp. 1481 - 1483, 2002.

[13] D. Stamatelos and A. Ephremides, "Spectral Efficiency and Optimal Base Placement for Indoor Wireless Networks," I EEE Journal on Selected Areas in Communications, vol. 14, pp. 651 - 661, 1996.

[14] K. Pahlavan and P. Krishnamurthy, Principles of Wireless Networks: A Unified Approach: Prentice Hall, Inc., 2002.

[15] T. S. Rappaport, Wireless Communications: Principles and Practice, Second ed: Prentice - Hall, Inc, 2002.

[16] M. A. Panjwani, A. L. Abbott, and T. S. Rappaport, "Interactive Computation of Coverage Regions for Wireless Communication in Multifloored Indoor Environments," IEEE Journal on Selected Areas in Communications, vol. 14, pp. 420 - 430, 1996.

[17] M.J.D. Powell, UOBYQA: Unconstrained Optimization by Quadratic Approximation, Mathematical Programming, Series B, 92(3), pp.555582, 2002.

[18] A.M. Bagirov, Minimization Methods for one Class of Nonsmooth Functions and Calculation of Semiequilibrium Prices, In: A. Eberhard et al. (eds.) Progress in Optimization: Contribution from Australasia, Kluwer Academic Publishers, pp. 147175, 1999.

[19] A.M. Bagirov, A Method for Minimization of Quasidifferentiable Functions, Optimization Methods and Software, 17(1), pp. 31-60, 2002.

[20] Cisco, "EOL: Cisco Aironet 340 Series Client Adapters and Access Points," Cisco Systems, 2003.

[21] Cisco, "Cisco Aironet 1200 Series Access Point," Cisco Systems, 2003.

[22] IEEE, "IEEE 802.11, 1999 Edition (ISO/IEC 880211:1999), IEEE Standards for Information Technology - Telecommunications and Information Exchange between Systems - Local and Metropolitan Area Network - Specific Requirements - Part 11: Wireless LAN Medium Access Control (MAC) and Physical Layer (PHY) Specifications," 1999. 